\title{
Comparison of standardized bariums with varying rheological parameters on swallowing kinematics in males
}

\author{
Jacqueline Hind, MS, CCC-SLP, BRS-S $;^{1-2 *}$ Elizabeth Divyak, MS, CCC-SLP; ${ }^{1}$ Jill Zielinski, MS, CCC-SLP; $^{1}$ \\ Andrew Taylor, MD; ${ }^{2}$ Michael Hartman, MD; ${ }^{1-2}$ Ronald Gangnon, PhD; ${ }^{2}$ JoAnne Robbins, PhD, CCC-SLP, \\ BRS-S $^{1-2}$ \\ ${ }^{1}$ William S. Middleton Memorial Veterans Hospital, Madison, WI; ${ }^{2}$ University of Wisconsin-Madison, Madison, WI
}

\begin{abstract}
This study measured dose-response of a range of commercially available liquid barium materials designed for use in videofluoroscopic oropharyngeal swallowing assessments, particularly as they relate to the necessity of adding a thickening agent for swallow safety. A group of 25 adult males representing various medical diagnoses consented to participate, with 16 qualifying to complete a videofluoroscopic swallowing assessment with liquid barium materials of three viscosities (nectar: $300 \mathrm{cP}$, thin honey: 1,500 cP, thick honey: 3,000 cP). Outcome measures included airway invasion (Penetration-Aspiration score), postswallow residue, and patient preference. Penetration-Aspiration and residue scores did not significantly differ between thin honey and thick honey bariums. Significantly more severe airway invasion was observed with nectar boluses than with two levels of honey boluses $(p<0.001)$. Significantly more residue was observed in the oral cavity $(p<0.002)$ and valleculae $(p<0.001)$ with thin and thick honey bariums than with nectar barium. Thin honey was rated as "easy" or "average" to drink by $67 \%$ of subjects, compared with $54 \%$ for thick honey. This study supports the use of thin honey barium over thick honey barium during videofluoroscopic swallowing assessments because the two honey bariums were comparable in terms of airway protection and postswallow residue in the oropharynx and the thin honey was preferred by patients.
\end{abstract}

Key words: aspiration, barium, deglutition, dysphagia, fluids, geriatrics, radiology, residue, swallowing, videofluoroscopy.

\section{INTRODUCTION}

More than 47 percent of veterans receiving healthcare from the Department of Veterans Affairs (VA) are over age 65 . The number of veterans over age 85 is estimated to reach 1.4 million in 2012, a 167 percent increase since 2000 [1]. As the aging veteran population grows along with the U.S. population, dysphagia and associated health consequences (e.g., dehydration, malnutrition, pneumonia) are becoming national healthcare concerns. More than 270,000 veterans with dysphagia accounted for 350,000 visits and admissions to VA facilities annually [2]. One of the most common treatments for dysphagia is thickening liquids, thereby modifying swallowing physiology by altering fluid rheology [3].

While modifying textures is a common treatment technique implemented by clinicians to prevent aspiration of food and liquids into the airway, much debate surrounds

\footnotetext{
Abbreviations: $\mathrm{NIH}=$ National Institutes of Health, VA = Department of Veterans Affairs, VFSS = videofluoroscopic swallow study.

*Address all correspondence to Jacqueline Hind, MS, CCC-SLP, BRS-S; VAMC GRECC 11G, 2500 Overlook Terrace, Madison, WI 53705; 608-256-1901, ext 11337; fax: 608-280-7023. Email: jahind@wisc.edu

http://dx.doi.org/10.1682/JRRD.2011.09.0180
} 
unified terminology and standardized rheological properties [4]. Many efforts have been made, some combining sensory and rheological measurements, to quantify food and liquid texture categories [5]. However, without radiopaque diagnostic correlates to the food/liquid textures, the definitions are of limited usefulness in dysphagia management.

Identifying the optimal treatment strategy for a dysphagic patient begins with a comprehensive evaluation of swallowing function. The videofluoroscopic swallow study (VFSS) is the most frequently used instrumental assessment procedure to determine the nature and extent of oropharyngeal dysphagia. During the examination, patients swallow systematically designed bariums of varying rheological composition to provide information on swallowing function. Distinct from barium materials used for other radiographic procedures (e.g., upper gastrointestinal series), these dysphagia-specific bariums (Varibar $^{\circledR}$, Bracco Imaging S.p.A; Milan, Italy) were designed to be nonadhesive to the oropharyngeal mucosa in nondisabled swallowers. These "noncoating" bariums allow clinicians to correctly identify any postswallow residue as abnormal, which is clinically imperative because residue remaining in the oropharynx after the swallow increases a patient's risk for aspiration once respiration resumes after the swallow.

The VFSS is distinct from many other radiographic examinations because of its ability to not only diagnose dysphagia, but also to verify the immediate effectiveness of treatment strategies in order to generalize the results when recommending a diet. Thickened liquids are a frequent short-term intervention poststroke, keeping patients safe from respiratory infection and other dysphagia sequelae while additional interventions are initiated during recovery. A survey of VA acute facilities completed by the Office of Inspector General reported that speechlanguage pathologists recommended a downgrade in diet consistency 68 percent of the time and recommended that all liquids be thickened 46 percent of the time following abnormal swallowing evaluations [6]. In certain circumstances (e.g., neurodegenerative conditions such as dementia), thickened liquids are recommended as a longterm solution for safe hydration [3]. As many as 31 percent of residents in long-term care facilities receive altered diets, with an estimated 28 percent receiving thickened liquids [7-10]. Of those receiving thickened liquids, on average, 60 percent received "nectar/syrup" thick, 33 percent received "honey" thick, and 6 percent received "pudding/spoon" thick [10].

Findings from the largest National Institutes of Health (NIH)-funded, multisite, randomized clinical trial for dysphagia provide the highest level of evidence available to demonstrate the effectiveness of thickened liquids to prevent (1) liquid aspiration in the fluoroscopy suite during the VFSS (short-term) [11] and (2) pneumonia during a 3 mo follow-up period (long-term) [12]. That trial enrolled 742 subjects who underwent a VFSS using thin ( $<15 \mathrm{cP}$ ), nectar thick (300 cP), and honey thick (3,000 cP) bariums. Results indicated that significantly more patients aspirated thin liquid barium than nectar (68\% vs $63 \%$, respectively) or honey barium (68\% vs 53\%, respectively) [11]. However, significantly fewer patients rated honey barium as "easy" or "average" to swallow compared with the other levels of barium-a finding with important relevance for compliance when translated to treatment (e.g., dietary recommendations). Moreover, the 3 mo follow-up of subjects randomly assigned to use an intervention revealed that, of those hospitalized for pneumonia, subjects drinking thick honey liquids $(3,000 \mathrm{cP})$ were hospitalized an average of $2 \mathrm{wk}$ longer than subjects drinking nectar liquids (median $=18$ vs $4 \mathrm{~d}$, respectively) [12]. The thick honey barium may be more challenging to safely clear from the airway because the force required to move material, in this case ejecting from the airway, increases with higher viscosity and yield stress [13].

Liquid barium with an intermediate viscosity of 1,500 cP (Varibar Thin Honey, Bracco Imaging S.p.A.) specifically designed for dysphagia evaluation has become available to clinicians in the last several years. The goal of the current project was to measure the dose-response of a range of systematically designed barium materials used for dysphagia diagnosis and treatment planning so that radiologists, speech-language pathologists, and other medical professionals can make informed decisions regarding the safest barium materials to use during VFSSs.

\section{MATERIALS AND METHODS}

Twenty-five male subjects referred for a standard clinical VFSS at the William S. Middleton Memorial Veterans Hospital consented to participate. Each VFSS was completed using a Siemens Model AXIOM Sireskop SD (Munich, Germany) recording at 30 frames per second. 
Images were recorded using a KayPENTAX Digital Swallowing Workstation (Lincoln Park, New Jersey).

Potential subjects were given three $3 \mathrm{~mL}$ boluses of Varibar Nectar (300 cP) administered in a teaspoon by the clinician and self-administered amounts from a cup. If aspiration was observed with this nectar barium, subjects subsequently were administered in random order three $3 \mathrm{~mL}$ boluses of Varibar Thin Honey $(1,500 \mathrm{cP})$ and three $3 \mathrm{~mL}$ boluses of Varibar Thick Honey (3,000 cP) barium administered in a teaspoon by the clinician and self-administered amounts from a cup. After each bolus type, subjects rated the fluid as "easy," "average," or "difficult and unpleasant" to drink.

Each swallow was scored using the Penetration/Aspiration Scale, an 8-point scale indicating the degree of airway invasion as well as the patient response to airway invasion. Higher scores (e.g., 8) signify more severe dysphagia as indicated by deeper airway invasion and/or diminished ability or effort to expectorate [14-15]. Postswallow barium residue was judged using a 3 -point scale $(0=$ no residue, 1 = coating of residue, 2 = pooling of residue) for each swallow at four locations (oral cavity, valleculae, posterior pharyngeal wall, and upper esophageal sphincter). The 3point residue scale is a subjective, nonvalidated but reliable scale that we have used in previously published studies [16].

The primary judge for this study was a speechlanguage pathologist who is a board-recognized specialist in swallowing and swallowing disorders. Interjudge reliability was completed by a second speech-language pathologist with $3.5 \mathrm{yr}$ of experience in quantification of VFSSs. Ten percent of the swallows were remeasured by the same judge and also by a second judge. Intrajudge reliability was 94 percent and interjudge reliability was 86 percent.

Penetration/Aspiration Scale scores and postswallow residue scores were modeled using linear mixed-effects regression models with age, bolus size (cup, $3 \mathrm{~mL}$ ), and bolus type (nectar: $300 \mathrm{cP}$, thin honey: 1,500 cP, thick honey: $3,000 \mathrm{cP}$ ) as fixed effects and subject as a random effect. Multiple imputation (using the aregImpute function in the Hmisc library in R) was used to account for missing data due to instrument malfunction. A nominal two-sided $p$-value of 0.05 was regarded as statistically significant.

\section{RESULTS}

\section{Subjects}

Twenty-five consecutively referred subjects were consented to participate in the study. Of those who consented, 16 qualified by aspirating nectar barium as visualized during a VFSS. Of those who qualified, all were males between the ages of 55 and 91 (mean: $77 \mathrm{yr}$ ). Twenty-five percent $(n=4)$ had a diagnosis of head or neck cancer/trauma, 13 percent $(n=2)$ had a diagnosis of cerebral vascular accident, 18 percent $(n=3)$ had a diagnosis of other neurologic disease, and 44 percent $(n=7)$ had a diagnosis of other medical condition (i.e., renal failure, pneumonia, infection).

\section{Penetration/Aspiration}

Penetration/Aspiration scores did not significantly differ between the thin honey $(1,500 \mathrm{cP})$ and thick honey $(3,000 \mathrm{cP})$ bariums $(p=0.66)$. A comparison between nectar $(300 \mathrm{cP}$ ) and both levels of the honey liquids (1,500 and 3,000 cP) revealed higher (i.e., more severe) Penetration/Aspiration scores with nectar boluses $(p<$ 0.001) (Figure 1). Higher Penetration/Aspiration scores were found with patient-controlled boluses from a cup than with the $3 \mathrm{~mL}$ boluses from a spoon $(p=0.01)$ with all barium types combined.

\section{Postswallow Residue}

There was no difference in residue scores between the thin honey and thick honey bariums at any location.

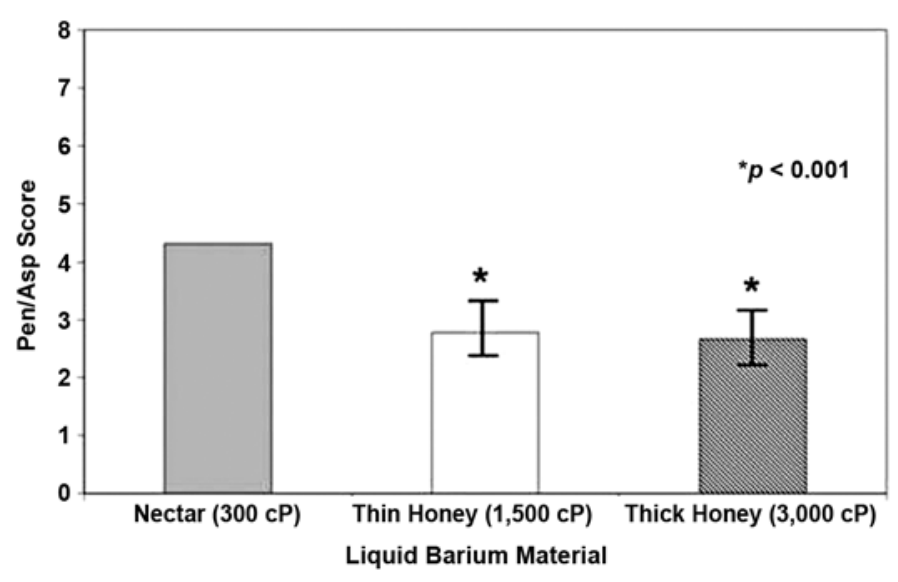

Figure 1.

Comparison of mean Penetration/Aspiration Scale (Pen/Asp) scores in fluids of $300,1,500$, and 3,000 cP. Higher Pen/Asp scores represent more severe airway invasion. 
However, significantly more residue was observed in the oral cavity $(p=0.002)$ and valleculae $(p<0.001)$ with both the thin and thick honey bariums than with the nectar barium (Figure 2). More residue was observed in the oral cavity, valleculae, and posterior pharyngeal wall with the cup boluses than with the $3 \mathrm{~mL}$ boluses ( $p<$ $0.001, p=0.007$, and $p=0.03$, respectively).

\section{Patient Preference}

Fifteen subjects verbally rated each type of barium during the VFSS as easy, average, or difficult and unpleasant to swallow. One subject was unable to answer the questions because of hearing loss. Subjects rated the nectar barium as average more often than the 1,500 or $3,000 \mathrm{cP}$ honey bariums ( $60 \%$ vs $40 \%$ and $27 \%$, respectively). More subjects rated the thick honey $(3,000 \mathrm{cP})$ to be difficult and unpleasant compared with the thin honey barium (1,500 cP) (46\% vs 33\%, respectively) (Table).

\section{DISCUSSION}

Results from this study indicate that during the VFSS, thin honey barium $(1,500 \mathrm{cP})$ and thick honey barium $(3,000 \mathrm{cP})$ did not significantly differ in terms of airway invasion or postswallow residue. These results are of great clinical relevance in light of published data indicating that patients who aspirated the more viscous $3,000 \mathrm{cP}$ fluids and contracted pneumonia had hospital stays three times longer than those who aspirated the less viscous nectar fluid [12]. Longer hospital stays may have been precipitated by the 3,000 cP fluid being more viscous and having a higher yield stress and, therefore, being more difficult to clear from the airway than less viscous liquids [13].

While managing airway invasion during the VFSS is a goal and recommending optimal beverages in terms of rheological parameters is one part of a treatment plan that may

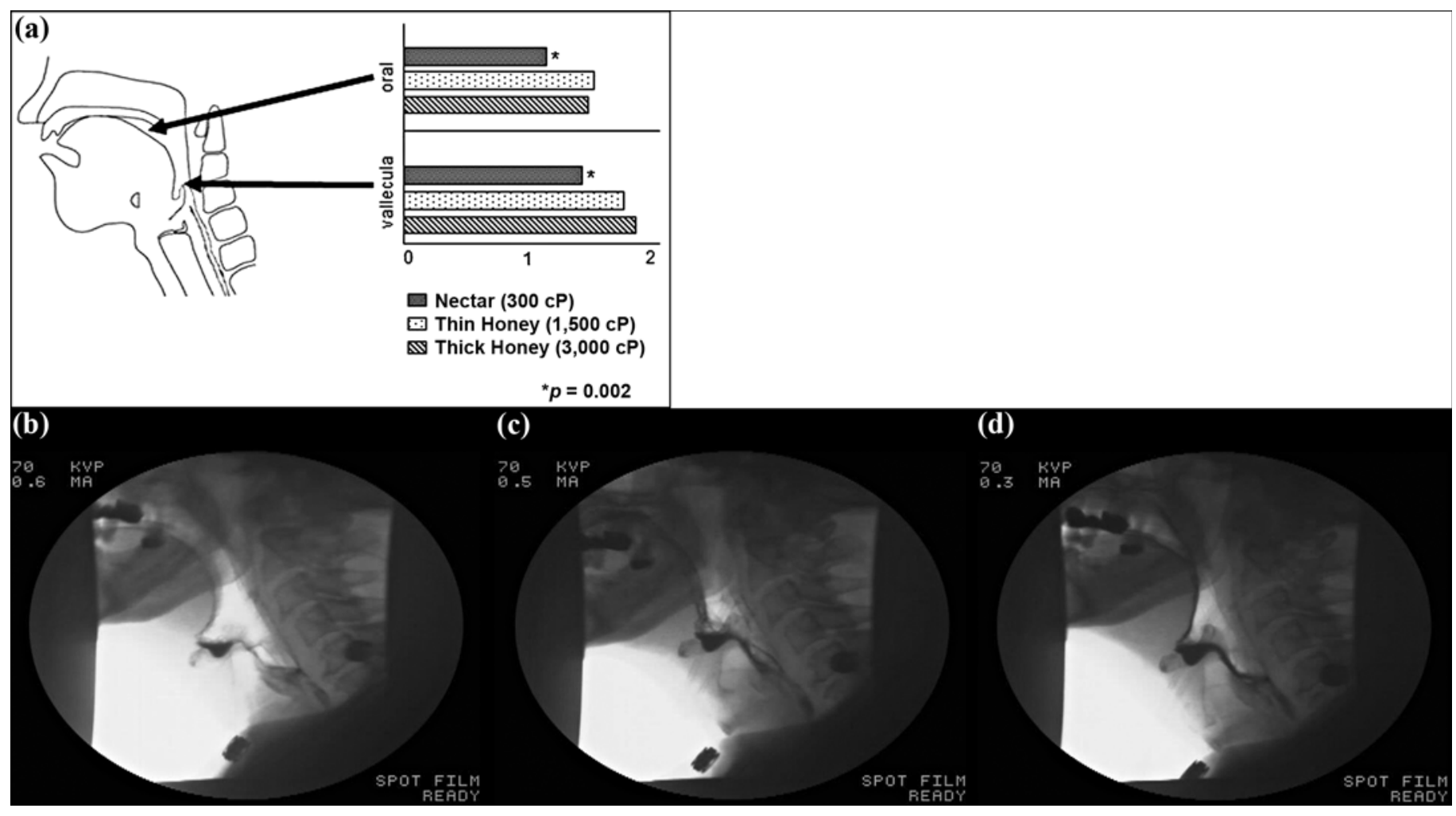

Figure 2.

(a) Comparison of mean postswallow residue scores at two locations in oropharynx (oral cavity and valleculae) with fluids of 300 , 1,500, and 3,000 cP. Higher scores represent more residue. ( $0=$ no residue, $1=$ coating of residue, $2=$ pooling of residue). Comparison of postswallow residue in oropharynx with fluids of (b) $300 \mathrm{cP}$ (nectar), (c) 1,500 cP (thin honey), and (d) 3,000 cP (thick honey). Postswallow residue as illustrated by select image frames from videofluoroscopy. Note increase of residuals on thin and thick honey boluses compared with nectar. 
Table.

Patient preference ratings for swallowing fluids of varying viscosity $(n=15)$.

\begin{tabular}{lccc}
\hline \multirow{2}{*}{ Patient Rating } & \multicolumn{3}{c}{ Patient Preference (\%) } \\
\cline { 2 - 4 } & $\begin{array}{c}\text { Nectar } \\
\mathbf{( 3 0 0} \mathbf{~ c P )}\end{array}$ & $\begin{array}{c}\text { Thin Honey } \\
\mathbf{( 1 , 5 0 0 ~ c P )}\end{array}$ & $\begin{array}{c}\text { Thick } \\
\text { Honey } \\
(\mathbf{3 , 0 0 0} \mathbf{~ c P )}\end{array}$ \\
\hline Easy & 20 & 27 & 27 \\
Average & 60 & 40 & 27 \\
Difficult/Unpleasant & 20 & 33 & 46 \\
\hline \hline
\end{tabular}

result from the VFSS findings or interpretations for dysphagia patients, the probability of totally eliminating aspiration is unlikely, even in relatively successful examinations and treatment plans. One could then postulate that the potentially more easily cleared, thin(ner) honey barium and subsequent "matched beverages" would result in shorter hospital stays for those individuals who aspirate and experience negative health status sequelae related to fluid intake. This hypothesis must be tempered, however, because nutritional fluids that exactly match the rheological properties of the barium products do not currently exist.

When assessing fluid preference in this study, 67 percent of subjects rated the thin honey as either easy or average to drink, compared with only 54 percent rating the thick honey positively. Since adherence with drinking thickened beverages, particularly the most thick, can be problematic, the thin honey fluid, which was identified as favorable, may be best to include in the VFSS because of improved therapeutic compliance, potentially preventing dysphagia-related health sequelae such as pneumonia.

\section{CONCLUSIONS}

Ideally, swallowing safety could be assessed with a multitude of liquids of varying rheological parameters during a radiographic examination to identify the safest and least restrictive diet for each patient. However, the goal of keeping radiation exposure to a minimum and, at times, the constraints of patient tolerance, limits clinicians to a few select contrast materials as representative samples of dietary beverages. Results from this study, as well as the largest NIH-funded clinical trial in the area of dysphagia, support the use of a finite set of optimal, representative standardized materials. Our study suggests using a thin honey $(1,500 \mathrm{cP})$ in place of the commonly used thick honey $(3,000 \mathrm{cP})$ barium during the radio- graphic assessment. This recommendation is based on comparable airway protection with both viscosity levels of honey bariums, the potential for easier airway clearance with the 1,500 cP barium, and the prospect of improved compliance when generalized to dietary fluid intake, which may include appealing beverages.

\section{ACKNOWLEDGMENTS}

\section{Author Contributions:}

Substantially contributing to study conception and design: J. Hind, A. Taylor, M. Hartman, R. Gangnon, J. Robbins.

Acquisition of data: J. Hind, E. Divyak, J. Zielinski, J. Robbins. Analysis and interpretation of data: J. Hind, E. Divyak, J. Zielinski, A. Taylor, M. Hartman, R. Gangnon, J. Robbins.

Drafting of article: J. Hind, A. Taylor, M. Hartman, R. Gangnon, J. Robbins.

Critical revision of article: J. Hind, E. Divyak, J. Zielinski, A. Taylor, M. Hartman, J. Robbins.

Providing final approval of version to be published: J. Hind,

E. Divyak, J. Zielinski, A. Taylor, M. Hartman, R. Gangnon,

J. Robbins.

Financial Disclosures: Dr. Robbins discloses a relationship with Swallow Solutions, LLC, which is dedicated to improving health status and quality of life for patients with swallowing disorders by focusing on clinical research in dysphagia. Swallow Solutions, LLC, had no role in study design, collection, analysis, data interpretation, report writing, or decisions to submit this manuscript.

Funding/Support: This material was based on work supported by the VA Veterans Health Administration, Office of Research and Development, Rehabilitation Research and Development Service (grant RRP 07-314). This is Geriatric Research Education and Clinical Center Manuscript 2011-02.

Additional Contributions: Many thanks to Abby Duane for manuscript preparation and insightful editing. Dr. Taylor is now with Virginia Commonwealth University in Richmond, Virginia.

Institutional Review: This study received approval from the University of Wisconsin-Madison Health Sciences Institutional Review Board and the VA Research and Development Committee. All subjects provided written informed consent.

Participant Follow-Up: The authors do not plan to inform participants of the publication of this study.

Disclaimer: The contents do not represent the views of the VA or the U.S. Government.

\section{REFERENCES}

1. U.S. Department of Health and Human Services. Statement by Judith A. Salerno, MD, at the National Institutes of Health Before the US House of Representatives [Internet]. Washington (DC): U.S. Department of Health and Human 
Services; 2004 Apr 29. Available from: http://www.hhs.gov/asl/testify/t040428c.html

2. Robbins J. The current state of clinical geriatric dysphagia research. J Rehabil Res Dev. 2002;39(4):vii-ix. [PMID:17638140]

3. Feinberg MJ, Knebl J, Tully J, Segall L. Aspiration and the elderly. Dysphagia. 1990;5(2):61-71. [PMID:2209101] http://dx.doi.org/10.1007/BF02412646

4. Penman JP, Thomson M. A review of the textured diets developed for the management of dysphagia. J Hum Nutr Diet. 1998;11:51-60. http://dx.doi.org/10.1046/j.1365-277X.1998.00079.x

5. Wendin K, Ekman S, Bulow M, Ekberg O, Johansson D, Rothenberg E, Stading M. Objective and quantitative definitions of modified food textures based on sensory and rheological methodology. Food Nutr Res. 2010;54:5134. [PMID:20592965]

6. Department of Veterans Affairs, Office of Inspector General. Healthcare inspection: evaluation of the management of patients with feeding and swallowing problems in Veterans Health Administration facilities. Report No. 0300494-110. Washington (DC): Department of Veterans Affairs, Office of Inspector General; 2006.

7. Groher ME, McKaig TN. Dysphagia and dietary levels in skilled nursing facilities. J Am Geriatr Soc. 1995;43(5): 528-32. [PMID:7730535]

8. Feinberg MJ, Ekberg O, Segall L, Tully J. Deglutition in elderly patients with dementia: findings of videofluorographic evaluation and impact on staging and management. Radiology. 1992;183(3):811-14. [PMID:1584939]

9. O'Gara JA. Dietary adjustments and nutritional therapy during treatment for oral-pharyngeal dysphagia. Dysphagia. 1990;4(4):209-12. [PMID:2119941] http://dx.doi.org/10.1007/BF02407267

10. Castellanos VH, Butler E, Gluch L, Burke B. Use of thickened liquids in skilled nursing facilities. J Am Diet Assoc. 2004;104(8):1222-26. [PMID:15281038] http://dx.doi.org/10.1016/j.jada.2004.05.203

11. Logemann JA, Gensler G, Robbins J, Lindblad AS, Brandt D, Hind JA, Kosek S, Dikeman K, Kazandjian M, Gramigna GD, Lundy D, McGarvey-Toler S, Miller Gardner PJ. A randomized study of three interventions for aspiration of thin liquids in patients with dementia or Parkinson's dis- ease. J Speech Lang Hear Res. 2008;51(1):173-83. [PMID:18230864] http://dx.doi.org/10.1044/1092-4388(2008/013)

12. Robbins J, Gensler G, Hind J, Logemann JA, Lindblad AS, Brandt D, Baum H, Lilienfeld D, Kosek S, Lundy D, Dikeman K, Kazandjian M, Gramigna GD, McGarvey-Toler S, Miller Gardner PJ. Comparison of 2 interventions for liquid aspiration on pneumonia incidence: a randomized trial. Ann Intern Med. 2008;148(7):509-18. [PMID:18378947]

13. Steffe J. Rheological methods in food process engineering. East Lansing (MI): Freeman Press; 1996.

14. Rosenbek JC, Robbins JA, Roecker EB, Coyle JL, Wood JL. A penetration-aspiration scale. Dysphagia. 1996;11(2): 93-98. [PMID:8721066] http://dx.doi.org/10.1007/BF00417897

15. Robbins J, Coyle J, Rosenbek J, Roecker E, Wood J. Differentiation of normal and abnormal airway protection during swallowing using the Penetration-Aspiration scale. Dysphagia. 1999;14(4):228-32. [PMID:10467048] http://dx.doi.org/10.1007/PL00009610

16. Hind JA, Nicosia MA, Roecker EB, Carnes ML, Robbins J. Comparison of effortful and noneffortful swallows in healthy middle-aged and older adults. Arch Phys Med Rehabil. 2001;82(12):1661-65. [PMID:11733879] http://dx.doi.org/10.1053/apmr.2001.28006

Submitted for publication September 28, 2011. Accepted in revised form March 20, 2012.

This article and any supplementary material should be cited as follows:

Hind J, Divyak E, Zielinski J, Taylor A, Hartman M, Gangnon R, Robbins J. Comparison of standardized bariums with varying rheological parameters on swallowing kinematics in males. J Rehabil Res Dev. 2012;49(9): 1399-1404. http://dx.doi.org/10.1682/JRRD.2011.09.0180

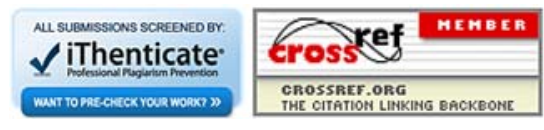

